Paper Number

\title{
The Use of Human Modeling Of EVA Tasks as a Systems Engineering Tool.
}

\author{
H. Charles Dischinger, Jr. \\ ED42, NASA/Marshall Space Flight Center, AL 35812 \\ c.dischinger@msfc.nasa.gov \\ (256) $544-9526(v),(256) 544-5551(f)$
}

Henry J. Schmidt

Tower II, The Boeing Company, Houston, TX 70559

henry.j.schmidt @ boeing.com

(281) 244-4601

\section{ABSTRACT}

Computer-generated human models have

been used in aerospace design for a decade. They have come to be highly reliable for worksite analysis of certain types of EVA tasks. In many design environments, this analysis comes after the structural design is largely complete. However, the use of these models as a development tool is gaining acceptance within organizations that practice good systems engineering processes. The design of the United States Propulsion Module for the International Space Station provides an example of this application. The Propulsion Module will provide augmentation to the propulsion capability supplied by the Russian Service Module Zvezda. It is a late addition to the set of modules provided by the United States to the ISS Program, and as a result, faces design challenges that result from the level of immaturity of its integration into the Station. Among these are heat dissipation and physical envelopes.

Since the rest of the Station was designed to maximize the use of the cooling system, little margin is available for the addition of another module. The Propulsion Module will attach at the forward end of the Station, and will be between the Orbiter and the rest of ISS. Since cargo must be removed from the Payload Bay and transferred to Station by the Canadarm, there is a potential for protrusions from the module, such as thruster booms, to interfere with robotic operations. These and similar engineering issues must be addressed as part of the development. In the implementation of good system design, all design solutions should be analyzed for compatibility with all affected subsystems. Human modeling has been used in this project to provide rapid input to system trades of design concepts. For example, the placement of radiators and avionics components for optimization of heat dissipation had to be examined for feasibility of EVA translation paths and worksite development. Likewise, the location of and mechanism for the retraction of thruster booms was partly driven by available Orbiter, robotic arm, and other module envelopes; worksite analysis was required for early assessment of task success. Since these trade studies included the EVA analysis as part of the decision criteria, the design had a high degree of assurance of EVA supportability from the outset. This approach contributes greatly to mission success.

\section{INTRODUCTION}

ExtraVehicular Activity (EVA) is widely regarded as an operational support to design and not a design solution. That is, 
operations are considered separate from the design process. The classical systems engineering approach, however, calls for allocation of resources and partitioning of responsibility among these resources. A simplistic example of this sort of allocation is the decision which results in having software, rather than a crewmember perform a simple calculation. This decision is often made unconsciously, but it can be tied to realistic systems engineering principles which dictate that decisions about resource allocation (in this example, crew mental processing and time vs. those available from an electronic computer) are part of the design process. In this approach, crew availability to participate in EVA should be considered a resource available to the systems engineer. In fact, this is the approach taken in International Space Station design projects. EVA is considered a resource available to systems engineers. Each design project is given an allocation at the outset (in number of EVA hours per year and during assembly), and the systems engineers are expected to stay within that allocation.

\section{Drawbacks and advantages of EVA} EVA is hazardous to crewmembers. The current designs of spacesuits provide considerably less protection against radiation and debris or micrometeoroid puncture than the Shuttle or ISS modules. Moreover, the risk of injury from hardware colliding with the EVA crew or from a torn suit is a constant concern. The suits are cumbersome, and thus hardware designed to be used by an EVA crewmember must include special features to accommodate EVA, These include large work and translation envelopes, specially-designed bolts, and strengthened hardware in the vicinity of translation paths. All of these add cost to a design. The activity itself is expensive. Crew time on any space mission is inherently costly, and system-level decisions should be made as to whether that cost is better expended on EVA than on other activities, such as science. A more direct cost than crew time comes from the consumables required for spacewalks. Suit parts, especially gloves, must be replaced on a regular basis due to wear, and the whole suit must be returned to earth and refurbished after six uses. Oxygen, water for drinking, and lithium hydroxide cannisters all must be changed or recharged each excursion. Suit batteries must be replaced. These consumables are not only inherently expensive due to development costs and flight certification, they constitute "upmass," at the current going rate of $\$ 20,000$ per kilogram.

Given these drawbacks, it becomes important to decide the circumstances under which EVA is to be used. Systems engineering approaches provide the framework for making these decisions, but they require rapid analyses of the complexity of the EVA tasks. Until recently (within the last ten years), no such rapid analytical tool has been available. Human modeling of EVA tasks provides for such input. This paper will explore the use of human modeling in systems decision making in an International Space Station design.

\section{The system.}

The ISS, like other satellites in low earth orbit, has a propulsion system. There are three basic maneuvers that this system must be able to accomplish. The first is attitude maintenance, or the holding of the vehicle in the proper orientation for solar panel functioning and for docking supply vehicles. The next capability is that of collision avoidance. There are many pieces of debris in or near the Station's orbit; the ISS must be able to maneuver to keep from being struck by one of these. The last and most dramatic operation is the reboost. Atmospheric drag causes reduction of altitude over time. The completed ISS would fall in a matter of months to a low enough orbit to burn, if it were not periodically boosted back up to a higher orbit.

The International Space Station is one-fault tolerant for propulsion. The propulsive capability is derived from the Russian Service Module Zvezda, which is in tum reliant on repeated refueling from Progress modules launched from the Baikonur Cosmodrome, which is not on Russian soil. While Russia does have another launch 
facility, it is only used for military launches, and all ISS launches are to be from Baikonur. In addition to this political uncertainty, the Russian Space Agency has recently had difficulty meeting supply schedules for Progress modules, due to funding challenges. While this system has worked flawlessly to date, a breakdown in any link from suppliers through on-orbit propulsive operations could threaten the ISS assets. The ISS Program has thus felt a second propulsion element was needed as a backup to the Russian capability. To this end, Boeing and NASA spent much of calendar years 1999 and 2000 attempting to develop a design for such a module. While the U.S. Propulsion Module is currently being redesigned, the effort to date can be instructive. There were several instances in which tradeoffs of EVA costs against system design constraints were made.

The U.S. Propulsion Module design concept called for a forward-facing (in the nominal ISS flight configuration) module which would serve as the primary docking port for the U.S. Orbiter when it visited the ISS (Figures 1 and 2). The design consists of a central passageway, surrounded by the exterior propulsion components, which include tanks, tubing, controllers, and thrusters (Figure 2). There are no EVA-servicable components in the propulsion system.

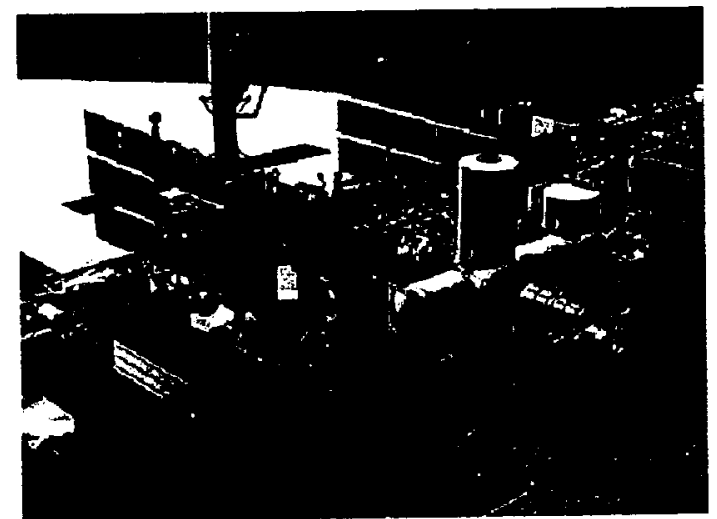

Figure 1. The forward end of ISS, with the Propulsion Module (lower right) in its nominal configuration, attached to Node 2. The ISS radiators are the accordionstructures pointed from the truss toward earth.

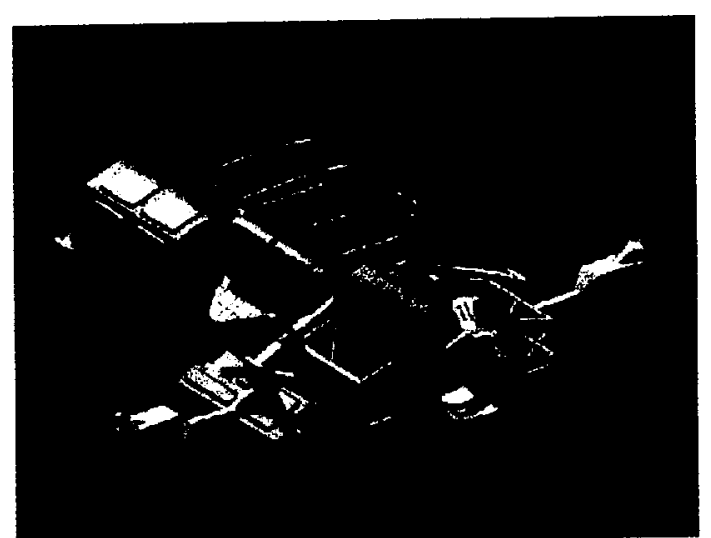

Figure 2. The U.S. Propulsion Module. The orientation is as in Figure 1, with the thrusters at the forward end of the module. The central circular structure is the Orbiter docking port, which leads to the interior crew passageway. The thruster booms are in the deployed positions.

\section{Design constraints}

This location for the module had several ramifications, both to the design of the module and to its operation. As a late addition to the ISS, it faced design constraints derived from the fact that many of Station's resources had previously been allocated. Normally, modules on ISS receive cooling by tying in to the distributed cooling system that uses the Station's large radiators to dissipate heat (Figure 1). However, this resource had already been apportioned. Thus, while the module could be physically connected to the Station refrigerant lines, there would be essentially no cooling capability available to it. All modules generate heat, from a variety of sources (crew activity, lights, computers, other electronic and electromechanical devices). This heat must be dissipated actively, or internal temperatures will quickly build up, making the module uninhabitable. In the absence of access to the Stationprovided cooling, the Propulsion Module thermal control team designed selfcontained radiators for the module.

Available physical envelopes are the volumes in which a module such as the Propulsion Module can be put into orbit and then operate. In this case, the module, like the remainder of the U.S.-provided components, is delivered by the Space 
Shuttle. The overall size constraint is driven by the ability of the Shuttle to enclose the launch package while getting it to orbit. The critical dimension $442 \mathrm{~cm}$. This is the dynamic envelope of the Payload Bay, which is the diameter of the Bay, minus the known "flex room," the allowance for the vibration of both the payload and the Shuttle without a collision. All items carried to Space Station by the Shuttle must be smaller than the dynamic envelope of the Shuttle. The operating envelope on-orbit is the volume available to it after other ISS components have been allocated the volumes they need to perform their functions. Of particular importance in this regard is the operation of the two versions of the Canadarm robot; one of these is attached to the Shuttle, and the other moves about on Station. Both are used to transfer components and supplies to ISS throughout the Station's lifetime. Thus, they must be allowed the envelopes in which to work, so that they can accomplish their missions.

\section{The decision-making process}

The factors which affect Space Station hardware and often result in EVA as a possible design solution include these and other constraints. The constraints limit the number of design solutions, but there is invariably more than one way to implement the design requirements. These solutions become part of the "trade space" which includes operational solutions such as EVA. The systems engineers must examine the design options and trade the relative importance of the constraints in order to arrive at the most suitable design. Evaluation of the contribution of the EVA constraints has become reliant on the use of spacesuited human models in the CAD system. This represents an important advance over older methods of EVA design. Even as recently as five to seven years ago, the primary method of assessing EVA issues was through neutral buoyancy simulation. This required that the structural design be largely complete, with the result that EVA issues were often resolved through operational workarounds or, if the issues were operationally insurmountable, major structural redesign. Either of these solutions tends to be costly. Nevertheless, systems engineers had no more reliable method of assessing the EVA design and thus gave little consideration to EVA issues when conducting trade studies.

\section{Interaction between constraints in the EVA design of the U.S. Propulsion Module}

Physical envelopes constitute among the most basic and confining of the design constraints, so they are incorporated into the design very early in the decision making process. In this manner, resolution of two conflicting requirements resulted in a decision with ramifications for the EVA design. The first of these was the need to fit the Propulsion Module into the dynamic envelope of the Shuttle's Payload Bay. The other was the operational requirement that the Propulsion Module be able to perform attitude control. This latter requirement resulted in the incorporation of deployable thruster booms into the design (see Figure 2). These booms would be deployed once the module was installed on Station, through a motor-driven mechanism. Evaluation of this configuration by the robotics community showed that the booms would interfere with certain Canadarm operations, and that one or both of them would need to be retracted several to possibly many times during the lifetime of the Station. Both for the initial deployment of the booms and for subsequent restow and redeploy (before and after robotics operations), EVA was required as a backup to the drive mechanism. That is, if the motor or some other component of the drive train failed to actuate, EVA would be required to assure Propulsion Module functionality, to allow robotics operations, or both. Thus, very early in the design process, the EVA modelers were called upon to assess candidate locations for the thruster boom attachment. The questions to be answered included whether it was possible to reach the task site and, once there, whether the requirements for astronaut work, reach, and visual envelopes could be met. While not a trade of EVA against some other design solution, this evaluation was critical to demonstrating a feasible design concept. It 
was found that for all of the suggested locations, a solution to the work envelope requirements could be met. However, the translation paths to these sites were not readily available. This was because the volume of the module almost completely filled the dynamic envelope of the Shuttle. The solution provided by the modelers was that the handrails needed to get to the drive override be left off the module for launch. Once the module was out of the Payload Bay, the handrails could be installed to provide a translation path to the task site. Figure 3 shows the translation path to the task site and a crewmember in position and driving the mechanism override. While the installation of handrails on-orbit adds EVA time, the task was considered acceptable. The trade in this case was to shrink the whole module $4 \mathrm{~cm}$ radially and allow ground installation or to perform the on-orbit installation. The structural (and thus, cost and schedule) impacts of the former were significant. A weighting factor in the trade was that access to the thruster booms was considered a contingency task.

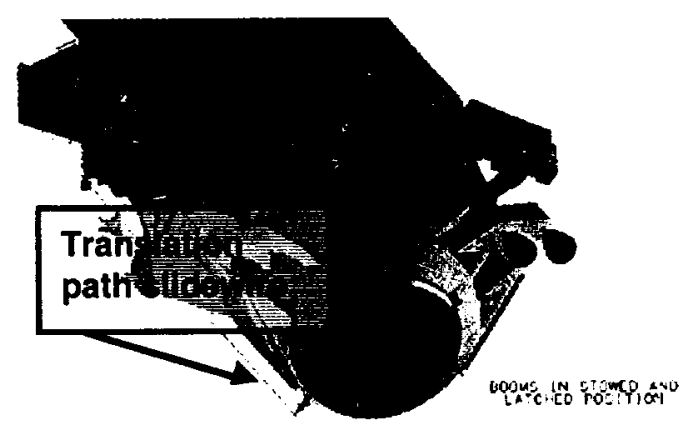

Figure 3. The analysis of the thruster boom mechanism override task; EVA crewmembers are in position to drive the override.

The thermal constraints imposed on the module resulted in some less-direct EVA design considerations. In one of these, EVA impact was a major component of the trade study. Since the cooling resources provided to other ISS components were mostly unavailable, the engineers for the Propulsion Module were responsible for solving the problem of the module's thermal dissipation without help from ISS. As part of the solution to this challenge, radiators were designed for the forward end of the module (see Figure 2). Initially, the proposal for the design called for quartz radiators. However, the location of the assembly was close to the primary EVA translation path between the Station and the Orbiter, when it was docked. While the EVA modelers could assure the radiators were far enough away from the handrails that the path requirements were met, the radiators were still within $122 \mathrm{~cm}$ of the handrails (see Figure 4). The requirement is that any structures within this distance of the handrails must be able to withstand the kick impacts of the Extravehicular Mobility Unit boot. Quartz does not meet this requirement, and in fact, might pose a serious hazard to the EVA crew from glass shards that might result from kicks. To avoid this hazard, the material was switched to a more durable silver-coated teflon. Thus, EVA model assessment allowed a decision (change in materials) to be made early that would have been very costly to implement late in the design cycle.

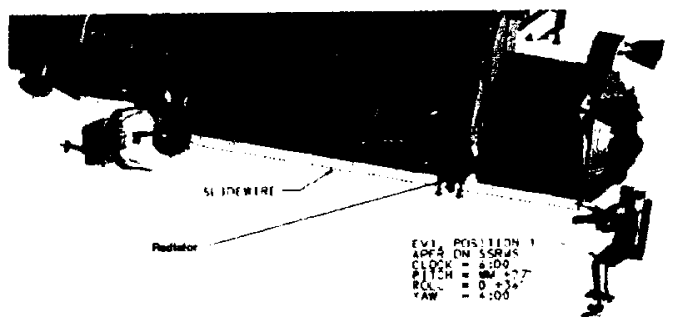

Figure 4. The Propulsion Module radiators are within $122 \mathrm{~cm}$ of the primary translation path, which is adjacent to the slidewire.

The decision to change materials for the radiators had other ramifications. The new material was not as efficient at exchanging heat as quartz, and it was not certain that even the quartz radiators could sufficiently dissipate the heat to allow the module to meet its requirement to not dump excess heat into the ISS atmosphere. A large portion of the heat created inside the module comes from the activity of the computers and other electronic components (avionics) during a propulsive maneuver. Propulsion would result in peaks of demand on the electronics, which would in turn require electric power and generate heat. In order to reduce this heat burden, the thermal team 
suggested that as many of the avionics boxes as possible be moved to the outside of the module. This solution created a problem for the ISS EVA community (the programmatic personnel responsible for overall ISS EVA operations). Almost all of the avionics components require maintenance; they must be replaced either periodically or upon failure. The problem for the EVA community was several-fold: 1. Could the boxes be arranged in an appropriate fashion so as to meet all EVA task site requirements? 2. Could an EVA excursion be mounted in sufficient time to allow replacement of critical avionics components? and 3 . Could these maintenance tasks be accomplished within the EVA allocation from Station (i.e., the number of hours available per year for Propulsion Module tasks)? The second question is not within the control of the designers, but the performance of the module is affected by it. The minimal time to mount an emergency EVA is twenty-four hours. The most severe emergency propulsive activity is the collision-avoidance maneuver, which would be required to avoid debris in the Station's orbit. It is believed that warning times for most potential debris impacts will be between one and two days. It was thus determined that this design solution was marginally safe, since a failure could probably be detected and repaired in time to accomplish such a maneuver. The EVA modelers were able to contribute real data to address questions 1 and 3. Their contribution to the design and analysis of the tasks showed that the worksite design could be made feasible (see Figure 5), and the number of hours required per year could be determined. The maintenance hours for this external avionics arrangement would require a $50 \%$ increase in the allocation from Station, for these tasks alone. If other EVA were to be required for the module, the number would go even higher.

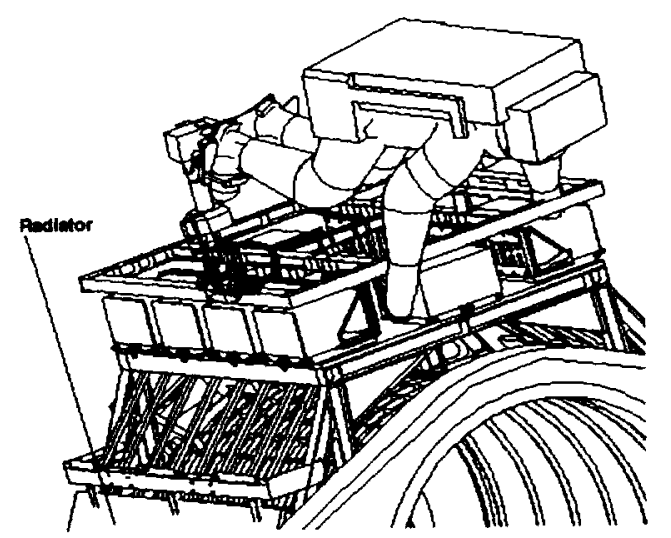

Figure 5. The layout of the avionics boxes on the exterior of the Propulsion Module; the forward end of the module (lower right, Figures $1 \& 2$ ) is shown. The example worksite analysis indicates the crewmember could be given appropriate task access.

The module EVA team performed analyses of all the tasks in less than a week, allowing this task information to be used in a trade of the thermal issues against those of EVA operations. While the task feasibility was proven, the design required an increase in yearly maintenance allocation from ISS. The considerations mentioned above about the risk and cost of EVA made this increase highly undesirable to the Program. This, combined with the uncertainty associated with the time required to accomplish a repair in the face of a potential debris collision, allowed the systems engineers to decide against the external placement of the avionics components.

Absent the use of the modeling tools, a rational basis for decision-making would have required that physical mockups be built and the tasks assessed in neutral buoyancy for feasibility and time requirements. This could not have been accomplished in the conceptual phase of the project, and delaying the decision would likely have resulted in redesign, which would have had undesirable cost and schedule effects. The alternative decision to find other ways to reduce the heat load were made. It was discovered that operational solutions (appropriate sequencing of computer use) would solve most or the challenges. Thus, the use of modern human modeling tools 
allowed decisions about resource allocations (here, EVA costs vs. thermal design costs) to be made on a logical basis.

\section{Conclusion}

In previous NASA programs, EVA has not been considered a resource. The missions which the agency desired to accomplish could easily absorb the costs and risks of EVA as part of the overall operation. The construction and maintenance of the International Space Station provides a new operating regime. The number of hours of EVA required for assembly is now estimated to be over 1000 . The costs associated with this amount of activity, and the risks to the crew have driven the ISS Program to consider EVA as a resource, for the purpose of making decisions about design solutions. The availability of human modeling in CAD systems has made this consideration possible. Prior to the emergence of this analytical tool, judgements in the early phases of design about task feasibility and time requirements were based primarily on personal experience. NASA has had very little experience with complex assembly tasks in EVA. The Propulsion Module project took advantage of the information available from this tool in considering the appropriate allocation of resources in its system design. The result, despite the halt of the current configuration of the project, is a design that intelligently utilizes EVA, in a cost-efficient and risk-aware manner. 